Public Abstract

First Name:Tasia

Middle Name:Marie

Last Name:Taxis

Adviser's First Name:William

Adviser's Last Name:Lamberson

Co-Adviser's First Name:

Co-Adviser's Last Name:

Graduation Term:FS 2015

Department:Animal Sciences

Degree:PhD

\title{
Title: Comparison of Rumen Microbe Taxa Among Wild and Domestic Ruminants
}

Microbes that inhabit the rumen provide a diverse set of enzymes that degrade ingested plant material. Without microbes, ruminants could not survive. Our research has focused on identifying microbial taxa associated with environment and phenotypic traits of host animals in order to improve livestock production and efficiency. Rumen contents were collected from a total of 48 animals from five host species, both wild and domestic, consuming either a concentrate- or forage-based diet. The objectives of this study were to analyze the distribution of microbial taxa among host species and identify microbial taxa associated with host diet, species, and domestication status. DNA was extracted from rumen content and sequenced for identification of microbial taxa present within each host animal. The distribution of microbial taxa was similar among host species. Each host species contained a few highly abundant microbial taxa and many microbial taxa in lower abundance. As host species were added to the analysis, the total number of microbial taxa identified increased and the number of microbial taxa common among all host species decreased, supporting the existence of a core microbial community. In this study we identified 66, 73, and 23 microbial taxa that differed among host diet, species, and domestication status, respectively. While on farm implications are far from being offered, studies of the microbial community add to the breadth of knowledge and identify microbes associated with performance and efficiency of livestock. 